\title{
Diversity, relative abundance and temporal spread of insects associated with Roselle (Hibiscus sabdariffa L.) at Makurdi, Nigeria
}

\author{
Lilian Dada Simon', Emmanuel Oludele Ogunwolu' ${ }^{2}$ Eunice Okoroafor ${ }^{2}$ and \\ Edache Ernest Ekoja ${ }^{2 *}$
}

Roselle, Hibiscus sabdariffa L., is cultivated extensively for food and income generation in Africa, but research on biotic constraints to its production has been scanty. A 48-plot ( $5 \mathrm{~m} \times 5 \mathrm{~m}$ wide each) field experiment laid in randomized complete block design was used to document the relative abundance (\% RA), diversity, richness, and temporal spread of insect species infesting the crop at Makurdi, Nigeria. The insects were collected from all parts of early- and latesown green-calyx ( $H$. sabdariffa var. sabdariffa) and red-calyx $(H$. sabdariffa var. altissima) Roselle shoots. About 101 species (81 herbivores, 18 predators, 1 parasitoid, and 1 pollinator) in 45 families and 8 orders were collected. Shannon's diversity index (2.1-2.4) and Margalef's richness index (8.3-10.0) indicate a rich diversity of species on the crop. However, evenness of species, measured by Buzas and Gibson's index, was low (0.1-0.41). The orders Coleoptera and Hemiptera accounted for $72.0 \%$ of the collection. Nineteen species were moderately ( $\geq 1 \mathrm{RA}<5 \%$ ) to highly abundant $(\mathrm{RA} \geq 10 \%)$ on the crop and among them Monolepta thompsoni Allard and Nisotra sjostedti Jac. were ubiquitous causing extensive leaf perforation all through the entire crop growth period. At the reproductive stage of growth, Dysdercus volkeri Fab., Oxycarenus hyalinipennis Costa and Earias sp. were the dominant insects causing fruit and seed damage. The frequency of occurrence and densities of $M$. thompsoni Allard, N. sjostedti Jac., D. volkeri Fab., O. hyalinipennis Costa and Earias sp. as well as their extensive damage, indicate that they are the key field pests of Roselle at Makurdi.

Keywords: Roselle, Insect species, Green-calyx, Red-calyx, Diversity, Richness, Temporal spread

'Department of Agronomy, Faculty of Agriculture, Federal University Gashua, Yobe State, Nigeria ${ }^{2}$ Department of Crop and Environmental Protection, Federal University of Agriculture, PMB 2373, Makurdi, Benue State, Nigeria

*Corresponding Author. Address: Department of Crop and Environmental Protection, Federal University of Agriculture, PMB 2373, Makurdi, Benue State, Nigeria; Email: ernestekoja@yahoo.com 


\section{INTRODUCTION}

Roselle (Hibiscus sabdariffa L.) is a multipurpose vegetable crop in the family Malvaceae. The leaves, calyces, seeds, and seed oil are regularly used as food, food condiment and colorant; as phytomedicine for treatment of diverse ailments; or as raw materials in pharmaceutical, cosmetic and paint industries (Alarcon-Aguilar et al 2007, El-Sheri and Sarwal 2007, Halimatul et al 2007, Alarcon-Alonso et al 2012, Ansari et al 2013, Olaniran et al 2013). Its cut flowers and decorative red stalks and fruits are thriving export commodities (Alegbejo et al 2003, Grubben et al 2004). A variety ( $H$. sabdariffa var. altissima) is an important fibre crop serving as a substitute to jute in paper industry (McClintock and Tahir 2004).

The crop is herbaceous, dicotyledonous, woody-based subshrub, annual/ biennial in nature, and widely adapted to a variety of tropical and sub-tropical climatic conditions (El Naim et al 2012, Kone et al 2018). It is believed to be native to Africa (Boulanger et al 1984, Gomez-Leyva et al 2008, Ankrah et al 2018). According to Sameer and Ali (2018), roselle plant could grow as tall as $2-2.5 \mathrm{~m}$ with three- to five-lobed leaves of about $8-15 \mathrm{~cm}$ length arranged alternately on the stems. The flowers could be yellow or red of about $8-10 \mathrm{~cm}$ in diameter having a stout fleshy calyx (1-2cm wide) at the base and could enlarge to $3-3.5 \mathrm{~cm}$ as the fruit matures. $H$. sabdariffa var. sabdariffa and $H$. sabdariffa var. altissima are the two major types of Roselle cultivated in Africa (Ankrah et al 2018). In Nigeria, the cultivation of Roselle straddles the diverse agro-ecological zones, and it is largely peasantry, polycultural, and purposively for food and income generation.

In spite of the crop's economic prospect, research on the biotic and environmental constraints to its production is limited. Insect pest infestation and damage have been identified as one of the major factors militating against the cultivation of roselle (Olaniran et al 2013). At different phenological stages, the plant is reported to be attacked by insect pests, and their dominance per plant in relation to other arthropods could be as high as $82.44 \%$ (Olaniran et al 2013, AbdelMoniem and Abd El-Wahab 2006). According to Simon et al (2018) the impact of insect pest infestation at vegetative and reproductive growth stages of Roselle could result in about $87.5 \%$ reduction in fresh calyx yield of the crop if synthetic control measures are not employed. Fasunwon and Banjo (2010) also identified Podagrica species as an important insect pest attacking both lamina of the foliage and matured leaves of the crop family. The insect was also implicated in the transmission of mosaic virus resulting in $20-50 \%$ yield reduction (Fajinmi and Fajinmi 2006). Roselle serves as habitat, oviposition or feeding site for different insect species on it; species richness and diversity and the pest status of the insects infesting the crop vary from one location to another. Documentations of insects associated with the crop in Nigeria are decades old (Daramola 1984, Dike 1992) and they emanated from a limited production area.

For the purpose of pest management, detection of new insect species, determination of the rate of species extinction and anthropogenic alteration of natural habitats, it is important to know the insect species, their relative abundance, diversity and richness on cultivated Roselle in a particular agroecology (Sisk et al 1994, Mirab-balou et al 2017). These insect-related variables were assessed in this pioneering study at Makurdi, Benue State, in the Nigerian Southern Guinea savanna using green- and red-calyx Roselle crops. 


\section{MATERIALS AND METHODS}

A 48-plot field experiment laid in randomized complete block design at the Agronomy Research Farm of Federal University of Agriculture, Makurdi (Latitude $07^{\circ} 45^{\prime}-07^{\circ} 50^{\prime} \mathrm{N}$, Longitude $08^{\circ} 45^{\prime}-08^{\circ} 50^{\prime} \mathrm{E}$ ) was used for enumeration and collection of insects on green-calyx ( $H$. sabdariffa var. altissima) and red-calyx $(H$. sabdariffa var. sabdariffa) Roselle planted early (June-September) and late (August-December) in 2016 cropping season. Each plot was $5 \mathrm{~m}$ long and $5 \mathrm{~m}$ wide; adjacent plots and replications were separated by $1 \mathrm{~m}$ and $2 \mathrm{~m}$ furrow, respectively. Plant stands were made comparable by thinning and replacing missing stands. Weeds were controlled manually and $100 \mathrm{~kg}$ of NPK (15-15-15) fertilizer was applied. Rainfall (cm) and Temperature $\left({ }^{\circ} \mathrm{C}\right)$ data were obtained from NIMET (Nigerian Meteorological Agency) substation at the Tactical Air Command, Nigerian Air Force, Makurdi, Nigeria.

Weekly visual enumerations of insects were made between 0700 and 1000 hours on plants enclosed by $1 \mathrm{~m} \times 1 \mathrm{~m}$ quadrat in rows 2 and 4 of each plot from 3 weeks after planting (WAP) to harvest (15WAP); the parts of the plant infested was noted before the insects were collected. The insects were killed in acetate jar and taken to the laboratory for sorting. At $50 \%$ flowering and $50 \%$ podding, five flowers and five pods were picked at random in rows 2 and 4 of each plot and opened to document number and species of insects found. Immature stages collected were reared to adult on appropriate food resource. Adult insects were identified at the Insect Museum of Ahmadu Bello University, Zaria, Nigeria.

The relative abundance of each species of insect was computed as:

$$
\mathrm{RA}(\%)=\mathrm{Ni} / \mathrm{N} \times 100 / 1
$$

Where: $\mathrm{Ni}=$ Number of individuals of a given species, and $\mathrm{N}=$ Total number of individuals of all species.

The species were categorized as:

$\begin{array}{ll}\text { Highly abundant } & \mathrm{Ar} \geq 10 \% \\ \text { Abundant } & \geq 5 \% \mathrm{Ar}<10 \% \\ \text { Moderately abundant } & \geq 1 \% \mathrm{Ar}<5 \% \text {, and } \\ \text { Scarce } & \mathrm{Ar}<1 \% \text { (Zaime and Gautier 1989). }\end{array}$

The frequency of occurrence of the insect species was computed as:

$$
\mathrm{C}=\mathrm{Pi} / \mathrm{P} \times 100 / 1
$$

Where: $\mathrm{Pi}=$ Number of occurrence of a particular species, and $\mathrm{P}=$ Total number of insects.

The species were classified as:

$\begin{array}{ll}\text { Ubiquist C } & 100 \% \\ \text { Constant } & 50 \% \geq C<100 \% \\ \text { Common } & 25 \% \geq C<50 \% \\ \text { By-catch } & 5 \% \geq C<25 \% \\ \text { Rare } & \mathrm{C}<5 \% \text { (Dajoz 2000). }\end{array}$


Species diversity was determined using Shannon's index:

$$
H^{\prime}=\sum_{i=1}^{\mathrm{s}} n_{\mathrm{i}} / N x \operatorname{lnn} n_{\mathrm{i}} / \mathrm{N}
$$

Where: $n_{i}=$ Number of individuals of the $i^{\text {th }}$ species in the sample; $N=$ Total number of all individuals in the assemblage; $S=$ Number of species in the assemblage.

Species richness was determined using Margalef's index:

$$
\mathrm{R}=(\mathrm{S}-1) / \ln (\mathrm{n})
$$

Where: $\mathrm{S}=$ Number of species; $\mathrm{n}=$ Number of individuals; In=Natural logarithm

Buzas and Gibson's Evenness:

$$
(E)=e^{H} / S
$$

Where: $e=$ Natural logarithm base; $H^{\prime}=$ Shannon index; $S=$ Number of species was used to determine species evenness. All indices were calculated using version 2.12 of the Paleontological Statistics Tool (Hammer et al 2001).

\section{RESULTS}

Of the 15,930 and 7,427 insects collected from the early and late crops, respectively, 102 species belong to 45 families and 8 orders were identified. Collection from the green-type $(13,376)$ exceeded that from the red-type Roselle by 3,395 insects. Eighty-one (81) of the species were phytophagous (Table 1) and 20 species were beneficial insects (Table 2). Coleopterous (especially Chrysomelidae and Lagriidae) and hemipterous (especially Pyrrhocoridae) insects constituted a high proportion $(72.0 \%)$ of the collections (Figure 1). Five species (Monolepta thompsoni Allard, Nisotra sjostedti Jac., Lagria villosa F., Oxycarenus hyalinipennis Costa and Dysdercus volkeri F.) were highly abundant; three species (Asbecesta cyanipennis Har., Carpophilus fumatus Boh., and Earias sp.) were abundant. Meanwhile, eleven species (Monolepta goldingi Bryant, Trichispa sericea GuérinMéneville, 1844, Bemisia tabaci Genn., Aphis gossypii Glover, Empoasca sp., Acrida bicolor Thunb., Aiolopus thalassinus Fab., Polistes spilophorus Schlett, Cheilomenes sulphurea Oliv., Exochomus flavipes Thunb. and Pheidole sp.) were moderately abundant. Among the three categories of abundant species, $M$. thompsoni, and $N$. sjostedti, were largely ubiquists (having $100 \%$ occurrence), while the other insect species with frequency of occurrence ranging from $>50 \%$ to $<100 \%$ were categorized as constant species. Table 3 shows a rich diversity of insects associated with Roselle at Makurdi going by Margalef's and Shannon's indices, respectively; however, Buzas and Gibson's evenness index was low indicating that only a few species dominated the ecosystem. Insect diversity and richness tended to be more on the green-calyx Roselle than on the red-calyx, and more in the earlythan in the late-sown crops. 
Diversity, relative abundance and temporal spread of insects

Table 1. Insect pests associated with early- and late-sown Roselle at Makurdi in 2016 cropping season

\begin{tabular}{|c|c|c|c|c|c|c|}
\hline \multirow{3}{*}{ Order } & \multirow{3}{*}{ Family } & \multirow{3}{*}{ Species } & \multicolumn{4}{|c|}{ Relative abundance (\%) } \\
\hline & & & \multicolumn{2}{|c|}{ Early-sown } & \multicolumn{2}{|c|}{ Late-sown } \\
\hline & & & $\begin{array}{l}\text { Green- } \\
\text { calyx }\end{array}$ & $\begin{array}{l}\text { Red- } \\
\text { calyx }\end{array}$ & $\begin{array}{l}\text { Green- } \\
\text { calyx }\end{array}$ & $\begin{array}{l}\text { Red- } \\
\text { calyx }\end{array}$ \\
\hline \multirow[t]{33}{*}{ Coleoptera } & Anthribidae & Araecerus sp. & 0.11 & 0.00 & 0.00 & 0.00 \\
\hline & Aphodiidae & Rhyssemus carinatipennis Per. & 0.02 & 0.03 & 0.07 & 0.06 \\
\hline & Buprestidae & Sternocera sp. & 0.70 & 0.47 & 0.23 & 0.13 \\
\hline & & Sphenoptera sp. & 0.00 & 0.00 & 0.05 & 0.06 \\
\hline & Carabidae & Drypta ruficollis Dej. & 0.01 & 0.03 & 0.00 & 0.00 \\
\hline & Chrysomelidae & Asbecesta cyanipennis Har. & 3.90 & 3.32 & 0.39 & 0.71 \\
\hline & & A. transversa Allard & 0.15 & 0.23 & 0.16 & 0.13 \\
\hline & & Aspidomorpha quinquefasciata Fab. & 0.02 & 0.03 & 0.00 & 0.00 \\
\hline & & Aulacophora africana Weise & 0.07 & 0.12 & 0.12 & 0.16 \\
\hline & & Lema sp. & 0.28 & 0.01 & 0.07 & 0.03 \\
\hline & & Luperodes quaternus Fairm & 0.00 & 0.03 & 0.12 & 0.00 \\
\hline & & Monolepta goldingi Bryant & 1.66 & 1.54 & 0.19 & 0.13 \\
\hline & & M. nigeriae Bryant & 0.71 & 0.68 & 0.12 & 0.13 \\
\hline & & M. thompsoni Allard & 27.52 & 27.84 & 17.68 & 20.19 \\
\hline & & Nisotra sjostedti Jac. & 23.77 & 24.95 & 14.60 & 13.68 \\
\hline & & Trichispa sericea Guer. & 0.99 & 0.96 & 0.81 & 1.09 \\
\hline & Coccinellidae & Epilachna chrysomelina Fab. & 0.00 & 0.00 & 0.05 & 0.00 \\
\hline & & E. hirta Thunb. & 0.00 & 0.00 & 0.00 & 0.03 \\
\hline & & E. similis Thunb. & 0.21 & 0.38 & 0.02 & 0.00 \\
\hline & Curculionidae & Alcidodes arcuatus Boh. & 0.00 & 0.01 & 0.00 & 0.00 \\
\hline & & A. senex Sahl. & 0.41 & 0.22 & 0.21 & 0.23 \\
\hline & & Apion sp. & 0.07 & 0.03 & 0.00 & 0.00 \\
\hline & & Baris sp. & 0.06 & 0.04 & 0.00 & 0.00 \\
\hline & & Cylas puncticollis Boh. & 0.03 & 0.04 & 0.00 & 0.03 \\
\hline & & Diacoderus sp. & 0.13 & 0.09 & 0.00 & 0.00 \\
\hline & & Siderodactylus sagittarius Sch. & 0.15 & 0.06 & 0.46 & 0.74 \\
\hline & Elateridae & Cardiophorus hoploderus Cand. & 0.01 & 0.03 & 0.21 & 0.13 \\
\hline & Hydrophilidae & Allocotocerus sp. & 0.08 & 0.01 & 0.00 & 0.00 \\
\hline & Lagriidae & Lagria villosa Fab. & 11.41 & 11.01 & 0.46 & 0.19 \\
\hline & Meloidae & Coryna sp. & 0.00 & 0.01 & 0.00 & 0.00 \\
\hline & & Mylabris vestita Reiche. & 0.01 & 0.03 & 0.74 & 0.87 \\
\hline & Nitidulidae. & Carpophilus fumatus Boh. & 4.27 & 2.72 & 0.02 & 0.06 \\
\hline & Scarabaeidae & Pachnoda sp. & 0.13 & 0.19 & 0.02 & 0.03 \\
\hline Dermaptera & Forficulidae & Diaperasticus erythrocephalus Oliv. & 0.01 & 0.00 & 0.35 & 0.16 \\
\hline \multirow[t]{7}{*}{ Hemiptera } & Aleyrodidea & Bemisia tabaci Genn. & 0.65 & 1.08 & 0.07 & 0.10 \\
\hline & Alydidae & Stenocoris elegans Blote. & 0.18 & 0.12 & 0.07 & 0.26 \\
\hline & & Tenosius sp. & 0.01 & 0.00 & 0.00 & 0.00 \\
\hline & Aphididae & Aphis gossypii Glover & 0.33 & 0.29 & 0.60 & 1.38 \\
\hline & & Myzus persicae Sulz. & 0.34 & 0.55 & 0.00 & 0.03 \\
\hline & Cicadellidae & Empoasca sp. & 1.08 & 1.37 & 0.23 & 0.23 \\
\hline & Coreidae & Anoplocnemis curvipes Fab. & 0.02 & 0.04 & 0.35 & 0.06 \\
\hline
\end{tabular}


Table 1 continued

\begin{tabular}{|c|c|c|c|c|c|c|}
\hline \multirow{3}{*}{ Order } & \multirow{3}{*}{ Family } & \multirow{3}{*}{ Species } & \multicolumn{4}{|c|}{ Relative Abundance (\%) } \\
\hline & & & \multicolumn{2}{|c|}{ Early-sown } & \multicolumn{2}{|c|}{ Late-sown } \\
\hline & & & $\begin{array}{l}\text { Green- } \\
\text { calyx }\end{array}$ & $\begin{array}{l}\text { Red- } \\
\text { calyx }\end{array}$ & $\begin{array}{l}\text { Green- } \\
\text { calyx }\end{array}$ & $\begin{array}{l}\text { Red- } \\
\text { calyx }\end{array}$ \\
\hline \multirow[t]{22}{*}{ Hemiptera } & Coreidae & Clavigralla tomentosicollis Stal. & 0.22 & 0.00 & 0.00 & 0.00 \\
\hline & & Cletus sp. & 0.00 & 0.00 & 0.00 & 0.10 \\
\hline & & C. albopunctatus Villier. & 0.01 & 0.00 & 0.07 & 0.03 \\
\hline & & C. notatus Thunb. & 0.04 & 0.06 & 0.05 & 0.13 \\
\hline & & Mirperus jaculus Thunb & 0.01 & 0.00 & 0.05 & 0.71 \\
\hline & Lygaeidae & Lygaeus rivularis Germ. & 0.07 & 0.09 & 0.00 & 0.00 \\
\hline & & Oxycarenus hyalinipennis Costa & 0.19 & 0.22 & 23.14 & 9.66 \\
\hline & Machaerotidae & Enderleinia bispina Schmidt & 0.06 & 0.06 & 0.00 & 0.00 \\
\hline & Miridae & Eurystylus sp. & 0.00 & 0.00 & 0.23 & 0.10 \\
\hline & & Megacoelum scutellare Popp. & 0.03 & 0.00 & 0.02 & 0.03 \\
\hline & Pentatomidae & Agonoscelis sp. & 0.03 & 0.00 & 0.00 & 0.00 \\
\hline & & A. erosa Westw. & 0.00 & 0.01 & 0.00 & 0.00 \\
\hline & & A. versicolor Fab. & 0.02 & 0.03 & 0.00 & 0.00 \\
\hline & & Aspavia hastator Fab. & 0.02 & 0.03 & 0.00 & 0.00 \\
\hline & & A. armigera Fab. & 0.01 & 0.00 & 0.02 & 0.00 \\
\hline & & Carbula pedalis Berg. & 0.11 & 0.01 & 0.02 & 0.03 \\
\hline & & Nezara viridula Linn. & 0.02 & 0.01 & 0.05 & 0.00 \\
\hline & Pseudococcidae & Planococcoides sp. & 0.02 & 0.01 & 0.00 & 0.00 \\
\hline & & Pseudococcus sp. & 0.00 & 0.00 & 0.05 & 0.00 \\
\hline & Pyrrhocoridae & Dysdercus volkeri Fab. & 14.63 & 15.07 & 27.77 & 35.74 \\
\hline & & Scantius clavimanus Fab. & 0.02 & 0.00 & 0.07 & 0.06 \\
\hline & Scutelleridae & Hotea sp. & 0.03 & 0.03 & 0.00 & 0.00 \\
\hline \multirow[t]{13}{*}{ Lepidoptera } & Arctiidae & Diacrisia sp. & 0.00 & 0.00 & 0.02 & 0.00 \\
\hline & & Metarctia sp. & 0.03 & 0.01 & 0.00 & 0.00 \\
\hline & Crambidae & Cirrhochrista sp. & 0.02 & 0.01 & 0.00 & 0.00 \\
\hline & & Palpita sp. & 0.01 & 0.00 & 0.00 & 0.00 \\
\hline & & Papyda sp. & 0.01 & 0.00 & 0.00 & 0.00 \\
\hline & Erebidae & Euproctis sp. & 0.00 & 0.00 & 0.25 & 0.26 \\
\hline & Nolidae & Earias sp. & 0.17 & 0.13 & 4.63 & 5.96 \\
\hline & & Hypodeva barbata Holland & 0.00 & 0.00 & 0.05 & 0.06 \\
\hline & & Negeta sp. & 0.00 & 0.00 & 0.02 & 0.00 \\
\hline & & Westermannia sp. & 0.00 & 0.00 & 0.02 & 0.00 \\
\hline & & W. agrapha Hamps. & 0.00 & 0.00 & 0.02 & 0.00 \\
\hline & Nymphalidae & Precis orythya Linn. & 0.00 & 0.00 & 0.46 & 0.00 \\
\hline & & P. sophia Fab. & 0.00 & 0.00 & 0.00 & 0.48 \\
\hline \multirow[t]{4}{*}{ Orthoptera } & Acrididae & Acrida bicolor Thunb. & 0.43 & 0.67 & 0.12 & 0.16 \\
\hline & & Aiolopus thalassinus Fab. & 0.74 & 0.80 & 0.30 & 0.61 \\
\hline & Pyrgomorphidae & Zonocerus variegatus Linn. & 0.02 & 0.01 & 0.00 & 0.10 \\
\hline & Tettigoniidae & Phaneroptera sp. & 0.04 & 0.04 & 0.37 & 0.35 \\
\hline
\end{tabular}


Diversity, relative abundance and temporal spread of insects

Table 2. Beneficial insects associated with early- and late-sown Roselle at Makurdi in 2016 cropping season

\begin{tabular}{|c|c|c|c|c|c|c|c|}
\hline \multirow{3}{*}{ Order } & \multirow{3}{*}{ Family } & \multirow{3}{*}{ Species } & \multirow{3}{*}{$\begin{array}{l}\text { Nature } \\
\text { of Insect }\end{array}$} & \multicolumn{4}{|c|}{ Relative Abundance (\%) } \\
\hline & & & & \multicolumn{2}{|c|}{ Early-sown } & \multicolumn{2}{|c|}{ Late-sown } \\
\hline & & & & $\begin{array}{l}\text { Green- } \\
\text { calyx }\end{array}$ & $\begin{array}{l}\text { Red- } \\
\text { calyx }\end{array}$ & $\begin{array}{l}\text { Green- } \\
\text { calyx }\end{array}$ & $\begin{array}{l}\text { Red- } \\
\text { calyx }\end{array}$ \\
\hline \multirow[t]{7}{*}{ Coleoptera } & Carabidae & Orthogonius sp. & Predator & 0.03 & 0.03 & 0.00 & 0.00 \\
\hline & Coccinellidae & Cheilomenes sulphurea Oliv. & Predator & 0.49 & 0.45 & 0.58 & 0.55 \\
\hline & & C. vicina Muls. & Predator & 0.10 & 0.12 & 0.00 & 0.00 \\
\hline & & Chilocorini sp. & Predator & 0.04 & 0.09 & 0.00 & 0.13 \\
\hline & & Exochomus flavipes Thunb. & Predator & 0.49 & 0.51 & 0.14 & 0.06 \\
\hline & Staphylinidae. & Paederus sabaeus Er. & Predator & 0.01 & 0.00 & 0.00 & 0.00 \\
\hline & Tenebrionidae & Eutochia pulla Er. & Predator & 0.02 & 0.00 & 0.02 & 0.03 \\
\hline \multirow[t]{5}{*}{ Hemiptera } & Pentatomidae & Macrorhaphis sp. & Predator & 0.01 & 0.03 & 0.00 & 0.00 \\
\hline & Reduviidae & Cosmolestes sp. & Predator & 0.00 & 0.00 & 0.00 & 0.06 \\
\hline & & Peprius nodulipes Signoret & Predator & 0.04 & 0.01 & 0.00 & 0.03 \\
\hline & & Rhynocoris sp. & Predator & 0.01 & 0.01 & 0.02 & 0.03 \\
\hline & & Tinna sp. & Predator & 0.00 & 0.00 & 0.00 & 0.10 \\
\hline \multirow[t]{5}{*}{ Hymenoptera } & Apidae & Eucera sp. & Pollinator & 0.00 & 0.01 & 0.07 & 0.00 \\
\hline & Braconidae & Iphiaulax sp. & Parasitoid & 0.29 & 0.25 & 0.19 & 0.42 \\
\hline & Formicidae & Camponotus sericeus Fab. & Predator & 0.17 & 0.17 & 0.62 & 1.00 \\
\hline & & Pheidole sp. & Predator & 0.36 & 0.36 & 0.16 & 0.42 \\
\hline & Vespidae & Polistes spilophorus Schlett. & Predator & 1.00 & 1.57 & 1.30 & 0.64 \\
\hline \multirow[t]{2}{*}{ Mantodea } & Mantidae & Sphodromantis sp. & Predator & 0.01 & 0.04 & 0.00 & 0.00 \\
\hline & Hymenopodidae & Pseudoharpax virescens Serv. & Predator & 0.28 & 0.33 & 0.19 & 0.13 \\
\hline Odonata & Libellulidae & Pantala flavescens Fab. & Predator & 0.00 & 0.00 & 0.12 & 0.19 \\
\hline
\end{tabular}

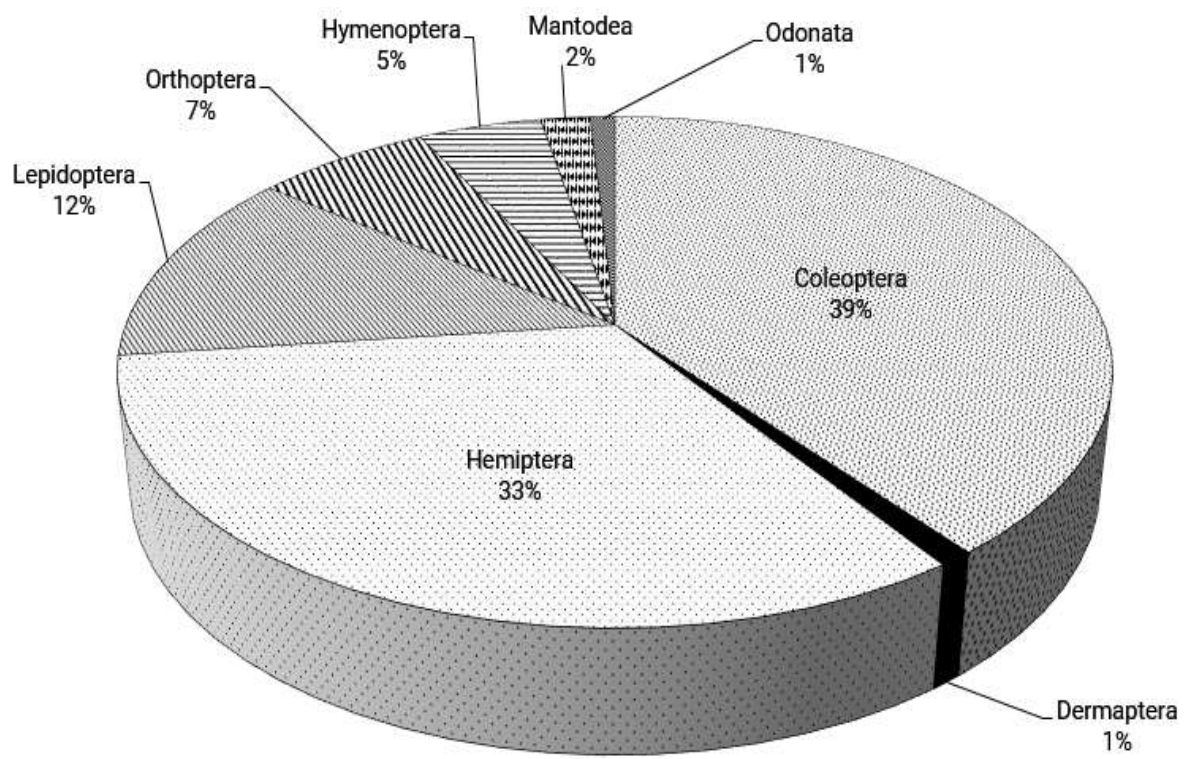

Figure 1. Relative abundance of insects orders associated with Roselle at Makurdi in 2016 cropping season 
Table 3: Insect species diversity and richness on Roselle at Makurdi in 2016 cropping season

\begin{tabular}{|c|c|c|c|c|c|c|c|c|}
\hline \multirow[b]{2}{*}{ Parameter } & \multicolumn{2}{|c|}{ Early-sown } & \multicolumn{2}{|c|}{ Late-sown } & \multicolumn{2}{|c|}{ Combined } & \multicolumn{2}{|c|}{ Combined } \\
\hline & $\begin{array}{l}\text { Green- } \\
\text { calyx }\end{array}$ & $\begin{array}{l}\text { Red- } \\
\text { calyx }\end{array}$ & $\begin{array}{l}\text { Green- } \\
\text { Calyx }\end{array}$ & $\begin{array}{l}\text { Red- } \\
\text { calyx }\end{array}$ & $\begin{array}{l}\text { Green- } \\
\text { calyx }\end{array}$ & $\begin{array}{l}\text { Red- } \\
\text { calyx }\end{array}$ & $\begin{array}{l}\text { Early- } \\
\text { sown }\end{array}$ & $\begin{array}{l}\text { Late- } \\
\text { sown }\end{array}$ \\
\hline No. of species collected & 82 & 71 & 64 & 63 & 96 & 89 & 88 & 72 \\
\hline №. of individuals collected & 9055 & 6875 & 4321 & 3106 & 13376 & 9981 & 15930 & 7427 \\
\hline Shannon-Wiener's Diversity index & 2.27 & 2.23 & 2.16 & 2.11 & 2.4 & 2.35 & 2.26 & 2.16 \\
\hline Buzas and Gibson's Evenness index & 0.12 & 0.13 & 0.13 & 0.14 & 0.11 & 0.12 & 0.11 & 0.12 \\
\hline Margalef's Richness index & 8.89 & 7.92 & 7.71 & 7.53 & 10 & 9.56 & 8.99 & 8.3 \\
\hline
\end{tabular}

All parts of the plants' shoot were colonized by insect pests (Table 4). Defoliators were preponderantly adult coleopterous insects among which $M$. thomsoni, $N$. sjostedti, A. cyanipennis and L. villosa were the abundant species (Figures 2 and 3 ). They were most abundant at the vegetative growth stage. At reproductive growth stage, both adult and immature stages of insects fed on the crop, the dominant species being $D$. volkeri, O. hyalinipennis and Earias sp. (Table 4). The number of insect species collected fluctuated during crop growth, but it increased phenomenally attaining a peak at 11 and 13 weeks after planting in the early- and the late-crop, respectively (Figures 4 and 5). Furthermore, Figures 6 and 7 show temporal spread of the insect species on Roselle. While infestation by $M$. thompsoni and $N$. sjostedti straddled the entire growth period in both cropping seasons, Earias sp., C. fumatus, L. villosa, A. gossypii, O. hyalinipennis, $D$. volkeri were limited to the reproductive growth stage of the crop. However, Empoasca sp. $B$. tabaci occurred only at the vegetative stage in the late-sown crops.

Table 4. Relative abundance, stage of insects, and the plant parts colonized on early- and late-sown Roselle at Makurdi in 2016 cropping season

\begin{tabular}{|c|c|c|c|c|c|}
\hline \multirow{2}{*}{ Species } & \multirow{2}{*}{ Status } & \multirow{2}{*}{ Stage } & \multirow{2}{*}{ Plant part attacked } & \multicolumn{2}{|c|}{ Relative abundance* } \\
\hline & & & & Early-sown & Late-sown \\
\hline Asbecesta cyanipennis Har. & Pest & Adult & Leaf & MA & $S$ \\
\hline Monolepta goldingi Bryant & Pest & Adult & Leaf & MA & s \\
\hline M. thompsoni Allard & Pest & Adult & Leaf & HA & HA \\
\hline Nisotra sjostedti Jac. & Pest & Adult & Leaf & HA & HA \\
\hline Trichispa sericea Guer & Pest & Adult & Leaf & $S$ & MA \\
\hline Lagria villosa Fab. & Pest & Adult & Leaf & HA & S \\
\hline Carpophilus fumatus Boh. & Pest & Adult & Flower & MA & S \\
\hline Bemisia tabaci Genn. & Pest & Adult & Leaf/Stem & MA & S \\
\hline Empoasca sp. & Pest & Adult & Leaf & MA & S \\
\hline Oxycarenus hyalinipennis Costa & Pest & Adult & Flower/Seeds & S & HA \\
\hline Dysdercus volkeri Fab. & Pest & Adult/Nymph & Flower/Fruit & HA & HA \\
\hline Aphis gossypii Glover & Pest & Adult/Nymph & Flower & S & MA \\
\hline Earias sp. & Pest & Larva/Adult & Flower/Fruit & $S$ & MA \\
\hline
\end{tabular}

$* S=S$ Sarce specie ( $<1 \%$ of total collection); $M A=$ moderately abundant species $(.21 \%$ but $<5 \%$ of total collection); $\mathrm{HA}=$ Highly abundant species ( $\geq 10 \%$ of the total collection) 
Diversity, relative abundance and temporal spread of insects

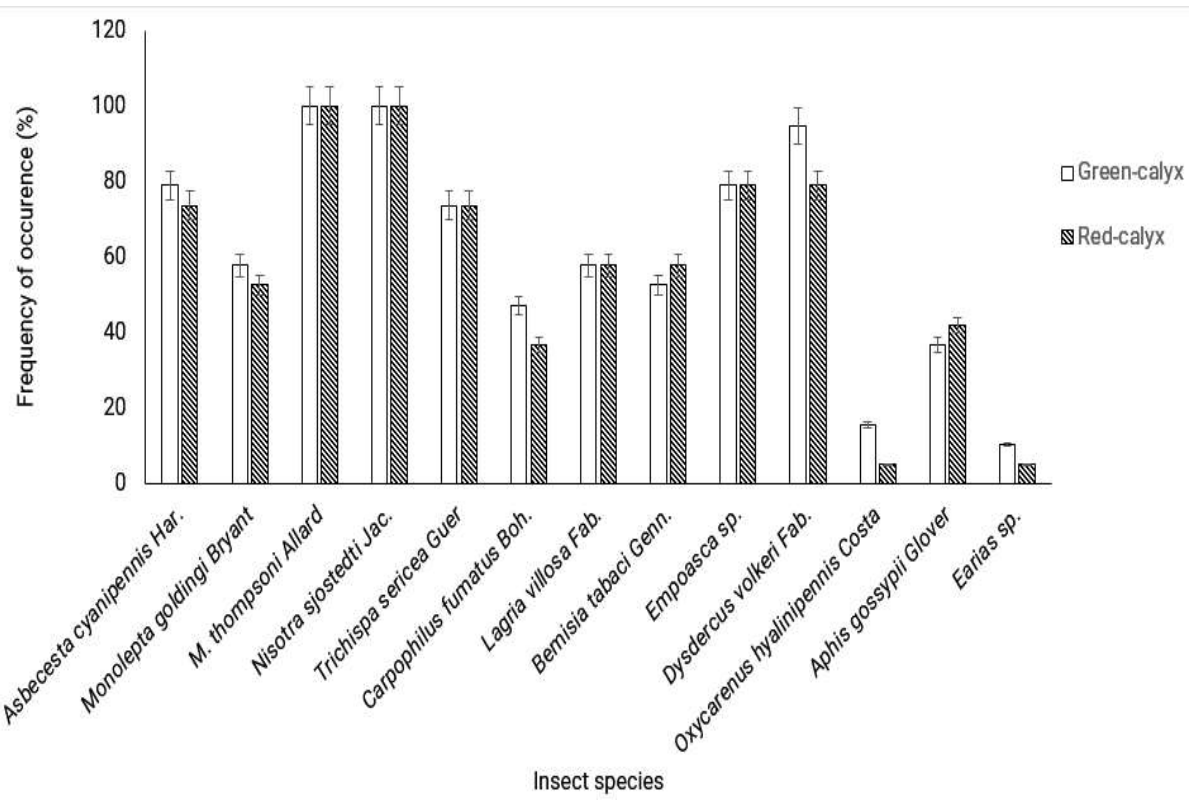

Figure 2. Frequency of insect occurrence on early-sown Roselle at Makurdi in 2016 cropping

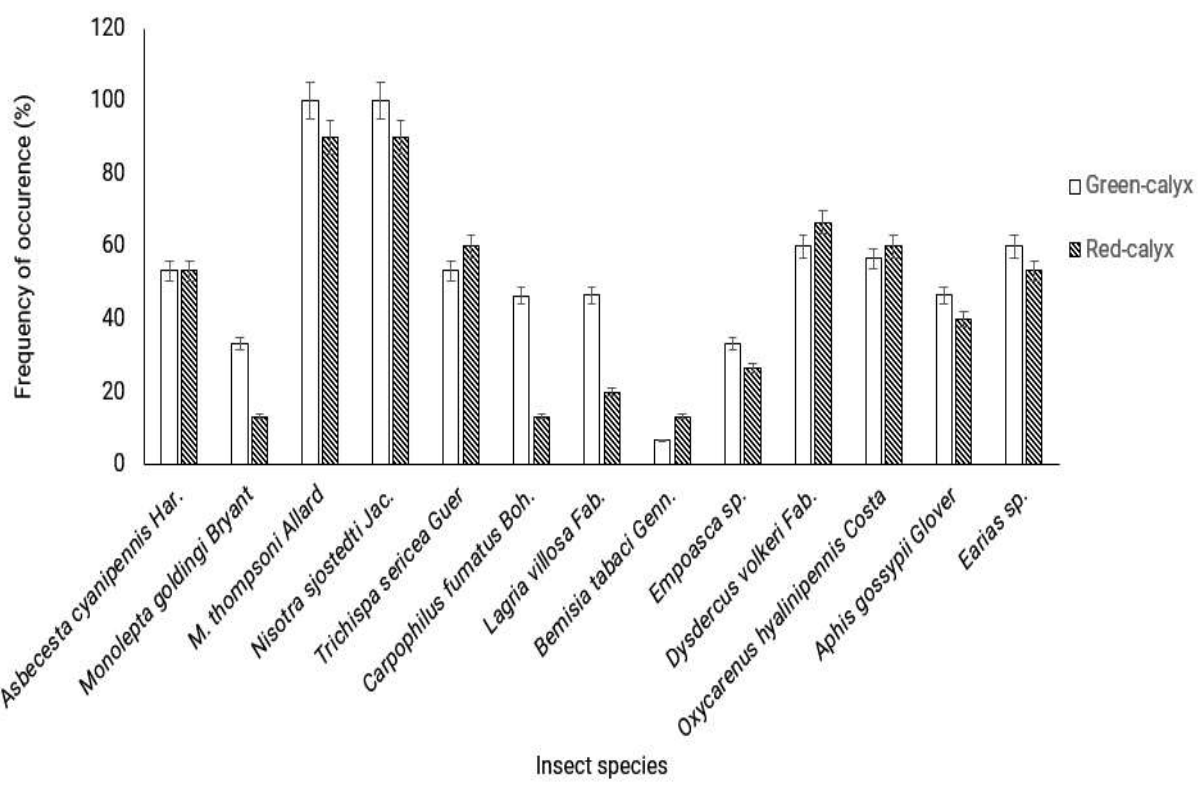

Figure 3. Frequency of insect occurrence on late-sown Roselle at Makurdi in 2016 cropping 
Simon et al

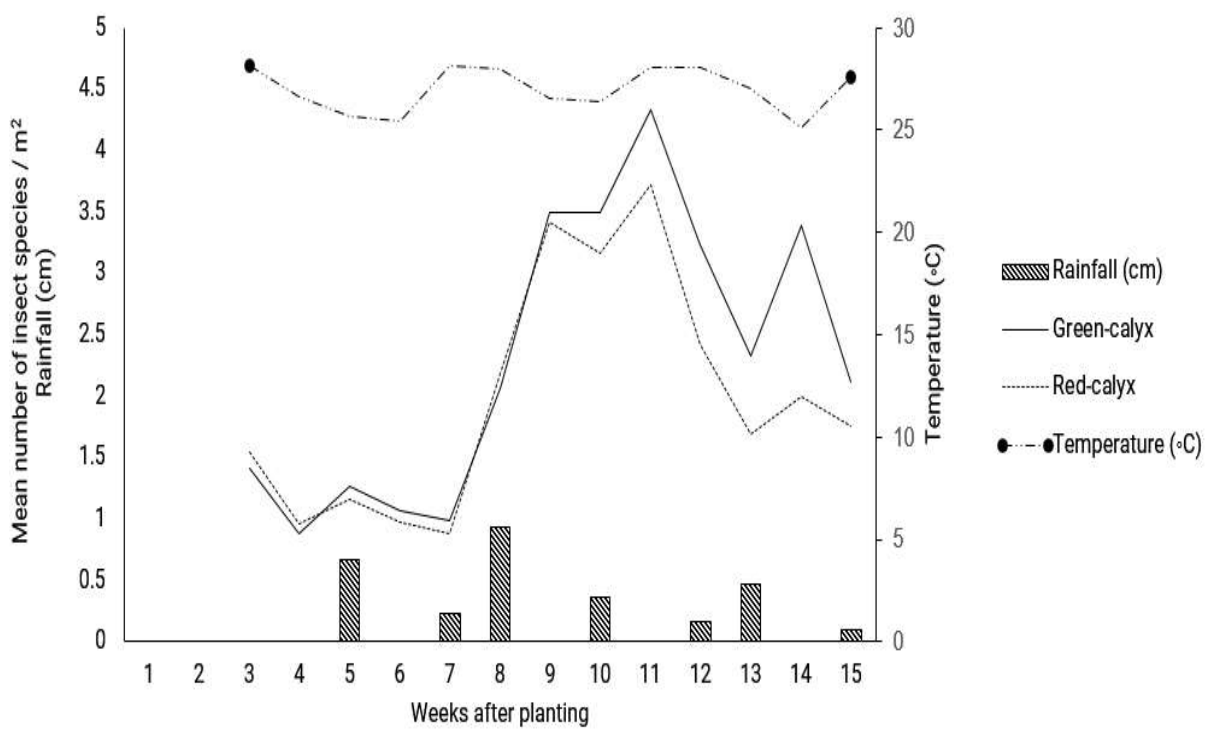

Figure 4. Weekly fluctuation in incidence of insect species infesting early-sown (June-September 2016) Roselle at Makurdi as influenced by rainfall and temperature of Makurdi, Nigeria (1-6 WAP=Vegetative stage; 7-15 WAP=Reproductive stage)

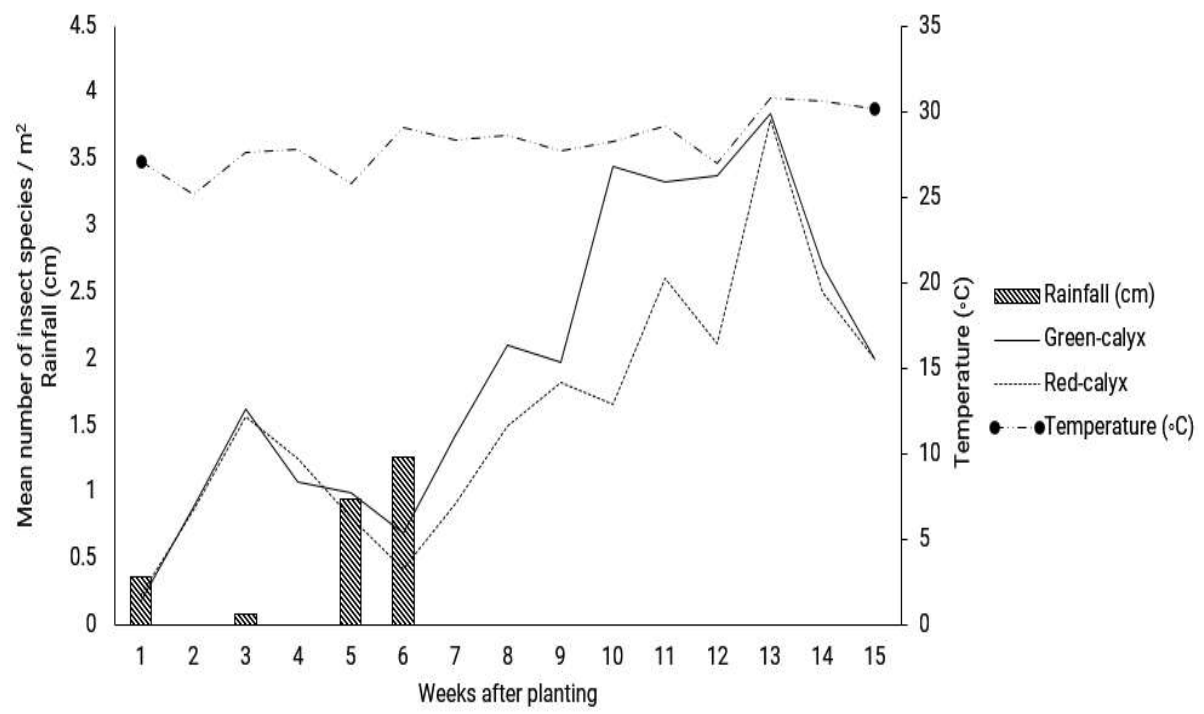

Figure 5. Weekly fluctuation in incidence of insect species infesting late-sown (August- December 2016) Roselle as influenced by rainfall and temperature of Makurdi, Nigeria (1-6 WAP=Vegetative stage; 7-15 WAP=Reproductive stage) 
Diversity, relative abundance and temporal spread of insects

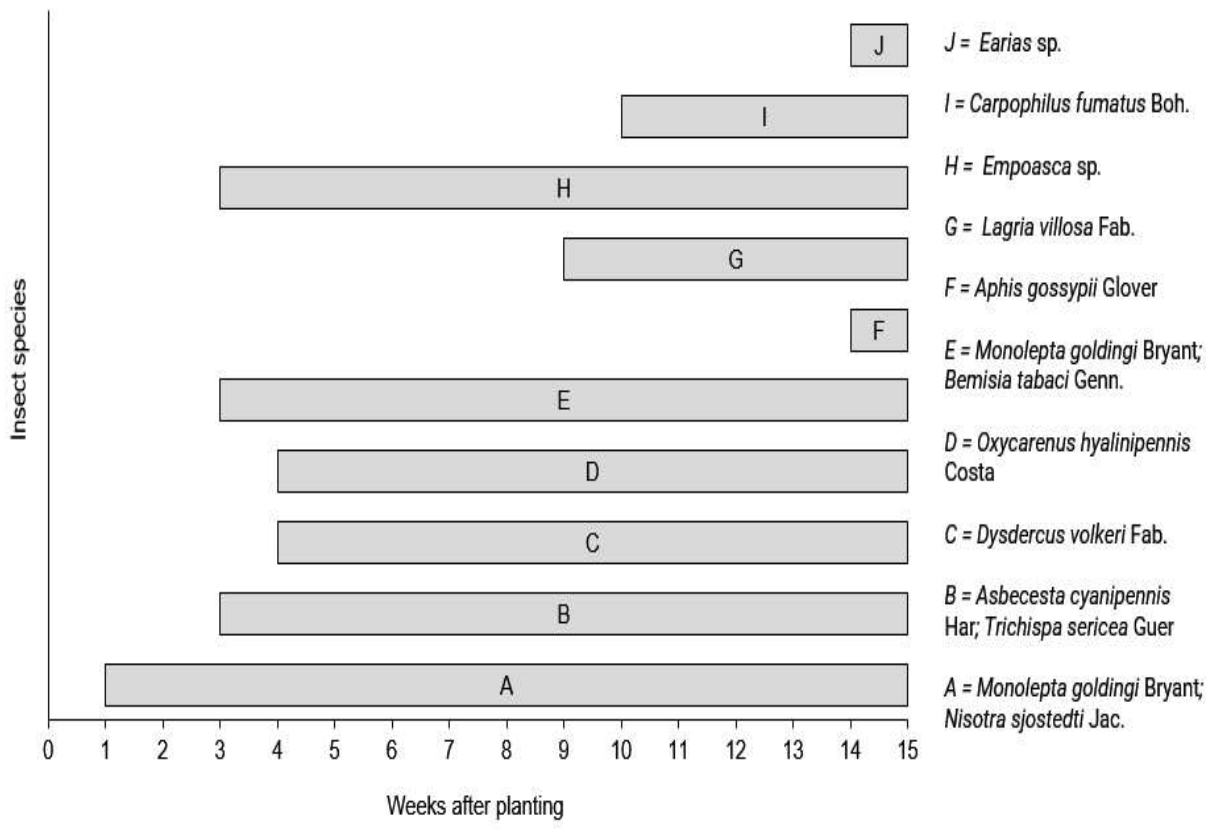

Figure 6. Temporal spread of insect species infesting early-sown Roselle at Makurdi in 2016 cropping season

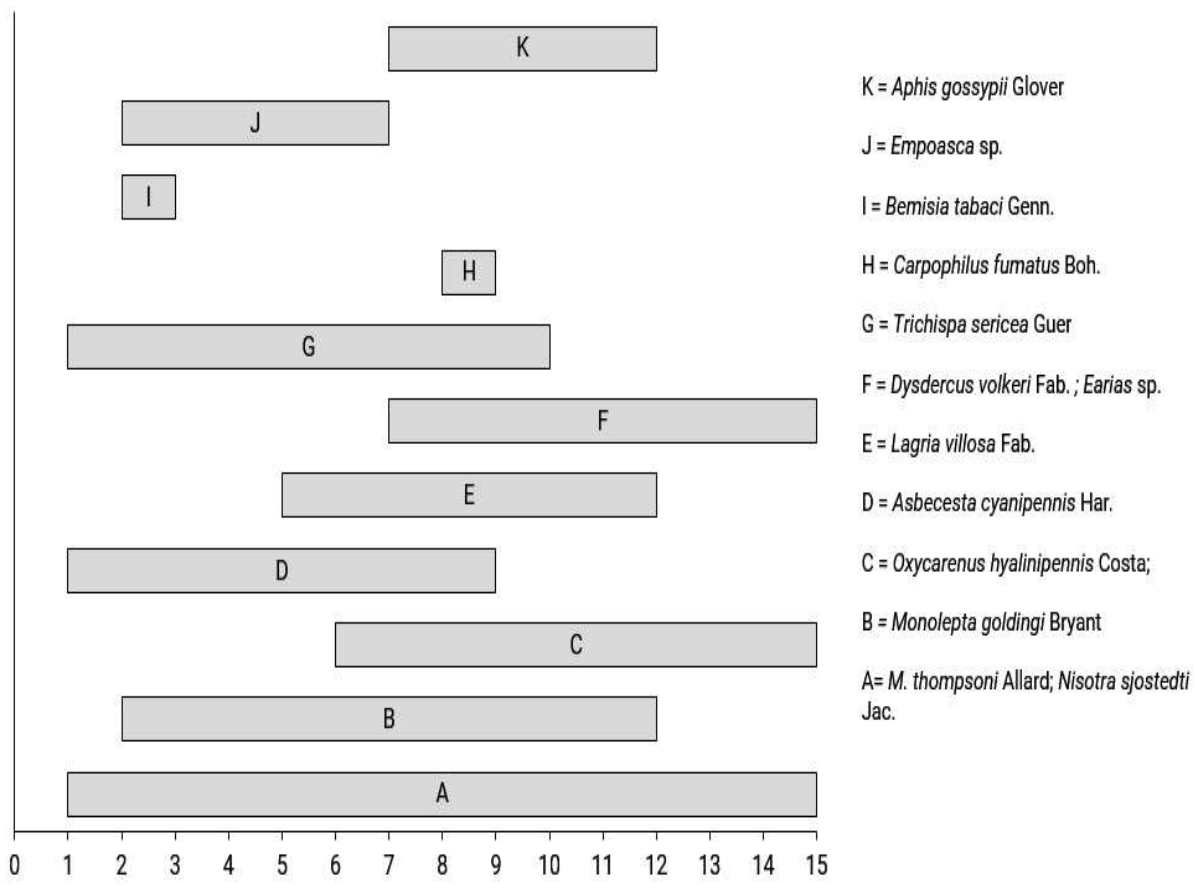

Figure 7. Temporal spread of insect species infesting late-sown Roselle at Makurdi in 2016 cropping season

(1-6 WAP=Vegetative stage; 7-15 WAP=Reproductive stage) 


\section{DISCUSSION}

Daramola (1984) observed 30 species of insects in 15 families and 4 orders infesting Roselle in farmers' fields in Southwestern Nigeria. In a study at Samaru, Dike (1992) recorded 11 species in 9 families and 3 orders. At Ogbomoso, Olaniran et al (2013) listed 5 insect pest species in 5 families and 4 orders. A far greater species diversity and richness have been documented in this paper. Nevertheless, many species reported elsewhere were not found at Makurdi. These include: Alcidodes grassirostris Thoms., Cheilomenes lunata F., Chrysolagria cuprina Thoms., C. nairobana Borch., Cryptocephalus obesus Suffr., Diplognata gagates F., Lobotrachelus incalidus Boh., Lycus semiamplexus Murr., Nematocerus acerbus Fst., Podagrica uniforma Jac., Pseudagrilus sophorae F., Silidius bennensis Pic., Stictoleis maculata F., Syagrus calcaratus F. [Coleoptera]; Clavigralla gibbosa Spin., Deraeocoris martini Puton, Drylocoris sp., Dysdercus superstitiosus F., Locris maculata F., Lygaeus festivus Thunb., Mirperus torridus Wstw., Nagusta sp., Oxycarenus gossypinus Dist., Rhinocoris bicolor F., (Hemiptera); Amascta flavicosta Ham. (Lepidoptera) (Daramola 1984, Dike 1992, Olaniran et al 2013). However, Cylas puncticollis Boh. (a known key insect pest of sweet potato, Ipomoea batatas) was observed on both green- and red-calyx Roselle plants in the study. This corroborates the findings of Malgwi and Onu (2013) who reported an instance of the insect feeding (nibbling and cutting holes or punctures) on various plant parts including young flowers, buds, and the soft apical growing points of five Malvaceae crop types (including Roselle) at Zaria, Nigeria.

$M$. thompsoni and $N$. sjosstedti, the frequently occurring and abundant coleopterous species, whose infestation straddled crop growth period, are classifiable as major pests at Makurdi given the extensive leaf perforation noticed particularly at the seedling and vegetative stage and the consequential adverse impact on plant vigour, growth, and yield (Ewete 1978, Clementine et al 2009). In Southwestern Nigeria, Daramola (1984) identified Chrysolagria cuprina Thoms., C. nairobana Borch, Podagrica uniforma Jac. and Syagrus calcaratus F. as the major seedling and foliage pests. In the present study, the dominant insects at the reproductive stage were $D$. volkeri, Earias sp. and 0 . hyalinipennis. This finding is consistent with the reports by Daramola (1984), Ewete and Osisanya (1984), Ottai et al (2004) and Abdel-Moniem et al (2011). Earias larvae perforated the fruits consuming its content and creating portals of entry for secondary invaders. Piercing and sucking of maturing seeds of Roselle by both $D$. volkeri and 0 . hyalinipennis culminated in seed weight loss and poor germination as reported by (Odhiambo 1957) on cotton in Uganda. The occurrence of piercing and sucking insects like Empoasca sp. and $B$. tabaci at the vegetative stage was not unexpected as these insects have been reported to cause significant damage to leaves and stems of roselle (Abdel-Moniem and El-Wahab 2006, El-Zoghby 2017).

Of the 20 beneficial insects documented, 18 were predatory species with $P$. spilophorus and $E$. flavipes as the dominant species in both the early-and late-sown crops. Abdel-Moniem and Abd El-Wahab (2006) had previously identified Polistes sp. as a dominant predator in Roselle fields in Egypt. The coccinelid beetles, $C$. sulphurea and $C$. vicina have been reported to prey upon aphids in Southwestern Nigeria (Daramola 1984). The impact of the predators and the only parasitoid found in this study (Iphiaulax sp.) was not determined. However, the ratio of beneficial 
insect to pest species was very low (1:4.1). This suggests a low impact level of natural biological control on the pest population in the location. If the aim is to promote biological insect pest control, repeated releases (augmentation) and implementation of measures that enhance the abundance or activity of the natural enemies, including manipulation of the crop microclimate (conservation), are needed to achieve an adequate level of insect pest control in the area.

\section{CONCLUSION}

Monolepta thompsoni Allard, Nisotra sjostedti Jac, Lagria villosa F., Oxycarenus hyalinipennis Costa and Dysdercus volkeri F. were the abundant phytophagous insect species. Natural enemies occurred at extremely low frequency and density, $P$. spilophorus was the dominant beneficial species encountered during this study.

It is suggested that feasibility of economically controlling the key field pests using resistant varieties, cultural techniques, physical barriers, semiochemical based technologies and, as a last resort, the use of selective chemicals which conserve beneficial insects in Roselle fields should be evaluated.

\section{ACKNOWLEDGMENTS}

The authors appreciate Mr. Musa Ishaku, of the Institute of Agricultural Research (IAR), Ahmadu Bello University, Zaria, Kaduna State, Nigeria who meticulously took time out to identify the insect samples.

\section{REFERENCES}

Abdel-Moniem ASH and Abd El-Wahab TE. 2006. Insect pests and predators inhabiting roselle plants, Hibiscus sabdariffa L., a medicinal plant in Egypt. Archives of Phytopathology and Plant Protection 39(1):25-32

Abdel-Moniem AS, Abd El-Wahab TE \& Fara NA. 2011. Prevailing insects in roselle plants, Hibiscus sabdariffa L., and their efficiency on pollination. Archives of Phytopathology and Plant Protection 44(3):242-252

Alarcon-Aguilar FJ, Zamilpa A, Perez-Garcia MD, Almanza-Perez JC, Romero-Nunez E \& Campos-Sepulveda EA. 2007. Effect of Hibiscus sabdariffa L. on obesity in MSG mice. Journal of Ethnopharmacology 114(1):66-71

Alarcón-Alonso J, Zamilpa A, Aguilar FA, Herrera-Ruiz M, Tortoriello J \& JimenezFerrer E. 2012. Pharmacological characterization of the diuretic effect of Hibiscus sabdariffa Linn. (Malvaceae) extract. Journal of Enthnopharmacology 139(3):751-756

Alegbejo MD, Abo ME \& Alegbejo JO. 2003. Current status and future potential of roselle production and utilization in Nigeria. Journal of Sustainable Agriculture $23(2): 5-16$

Ankrah NA, Tetteh AY, Nancy Coffie N \& Niagiah A. 2018. Characterization of roselle (Hibiscus sabdariffa L. var. altissima Wester) accessions in Northern Ghana by agro-morphological traits. Journal of Agricultural Science 10(9):64-75

Ansari M, Eslaminejad T, Sarhadynejad Z \& Eslaminejad T. 2013. An overview of the roselle plant with particular reference to cultivation, diseases and usage. European Journal of Medicinal Plants 3(1):135-145 
Boulanger J, Follin JC \& Bourely J. 1984. Les hibiscus textiles en Afrique tropicale, 1ère partie: Conditions particulières de production du kenaf et de la roselle (5th edn). Coton et Fibres Tropicales

Clementine LD, Malick NB, Koussao S \& Antoine S. 2009. Preliminary studies on incidence of insect pest on okra, Abelmoschus esculentus (L.) Moench in central Burkina Faso. African Journal of Agricultural Research 4(12):1488-1492

Dajoz R. 2000. Précis d'écologie. Dumond (7th edn), Paris

Daramola AM. 1984. The insect pest complex of the edible roselle, Hibiscus sabdariffa var. sabdariffa L., and the damage they cause in South Western Nigeria. Nigerian Journal of Entomology 5:62-69

Dike MC. 1992. Preliminary survey of insect pests of kenaf and roselle around zaria in the Northern Guinea Savanna of Nigeria. Nigerian Journal of Plant Protection 14:13-20. In Alegbejo MD, Abo ME \& Alegbejo JO (eds) Current Status and Future Potential of Roselle Production and Utilization in Nigeria. Journal of Sustainable Agriculture 23(2):5-16

El-Naim AM, Khaliefa EH, Ibrahim KA, Ismaeil FM \& Zaied MMB. 2012. Growth and yield of roselle (Hibiscus sabdariffa L.) as influenced by plant population in arid tropic of Sudan under rain-fed. International Journal of Agriculture and Forestry 2(3):88-91

El-Sherif $\mathrm{MH}$ and Sarwat MI. 2007. Physiological and chemical variations in producing roselle plant (Hibiscus sabdariffa L.) by using some organic farmyard manure. World Journal of Agricultural Science 3(5):609-616

El-Zoghby IRM. 2017. Studies on the impact of successive sprays with certain insecticides on whitefly and aphids infesting roselle plants and its yield in Aswan Governorate, Egypt. Middle East Journal of Applied Sciences 7(1):162167

Ewete FK. 1978. Insect species and description of damage caused on okra, Abelmoschus esculenthus (L.) Moench. East African Agricultural Forestry Journal 44(2):152-163

Ewete FK and Osisanya EO. 1984. Effect of various diets (seeds) on development, longevity and fecundity of the cotton seed bug, Oxycarenus gossypinus Distant (Heteroptera: Lygaeidae). Insect Science Applied Journal 6(4):543-545

Fajinmi AA and Fajinmi OB. 2006. Incidence of okra mosaic virus at different growth stages of okra plants (Abelmoschus esculentus L. Moench) under tropical condition. Journal of General and Molecular Virology 2(1):028-031

Fasunwon BT and Banjo AD. 2010. Seasonal population fluctuations of species on okra plant (Abelmoschus esculentus). Research Journal in Agriculture and Biological Sciences 6(3):283-288

Gomez-Leyva JF, Acosta LAM, Muraira IGL, Espino HS, Ramirez-Cervantes F \& Andrade-Gonzalez I. 2008. Multiple shoot regeneration of roselle (Hibiscus sabdariffa L.) from a shoot apex system. International Journal of Botany 4(3):326-330

Grubben GJ, Denton OA, Messiaen GM, Schippers RR, Lemmens RH \& Oyen LA. 2004. Plant resources of tropical Africa. Wageningen, Netherlands: Backhuys Publishers

Halimatul SM, Amin I, Mohd.-Esa N, Nawalyah AG \& Siti-Muskinah M. 2007. Protein quality of roselle (Hibiscus sabdariffa L.) seeds. ASEAN Food Journal $14(2): 131-40$ 
Hammer O, Harper DAT \& Ryan PD. 2001. PAST: paleontological statistics software package for education and data analysis. Palaeontologia Electronica 4(1):1-9

Kone D, Yapo SES, Abeda ZH, Kouadio OKS, Koffi E \& Kouakou TH. 2018. Effects of explant type and hormone combinations on callus induction at high production potential of phenolic compounds in roselle (Hibiscus sabdariffa var. sabdariffa). European Journal of Biotechnology and Bioscience 6(5):91-97

Malgwi MA and Onu I. 2013. Alternate host plants, hibernation sites and survival strategy of Cylas puncticollis Boh.: A new pest of cotton. Journal of Biology, Agriculture and Healthcare 3(2):9-22

McClintock NC and Tahir I. 2004. Hibiscus sabdariffa L. In Grubben GJH \& Denton OA (eds) Plant Resources of Tropical Africa 2. Vegetables. PROTA Foundation, Wageningen, Netherlands/Backhuys Publisher, Leiden, Netherlands

Mirab-balou M, Mahmondi M \& Tong X. 2017. Diversity of thrips species (Thysanura) in fruit orchards in Qazvin Province, Northwestern Iran. Journal of Crop Protection 6(3):363-373

NIMET (Nigerian Meteorological Agency). 2016. Annual weather bulletin of the Nigerian Meteorological Agency. Makurdi: Tactical Air Command, Nigerian Air Force

Odhiambo TR. 1957. The bionomics of Oxycarenus species (Hemiptera: Lygaeidae), and their status as cotton pests in Uganda. Journal of Entomological Society South Africa 20:235-249

Olaniran OA, Alao FO \& Adebayo TA. 2013. Control of foliage pests of roselle (Hibiscus sabdariffa I.) using plant extracts of Tephrosia vogelii and Azardiractha indica in Ogbomoso, Nigeria. Transnational Journal of Science and Technology 3(6):51-62

Ottai MES, Abdel-Moniem ASH \& El-Mergawi RA. 2004. Effect of variety and location on growth and yield components of roselle, Hibiscus sabdariffa L. and its infestation with the spiny bollworm Earias insulana (Boisd). Archives of Phytopathology and Plant Protection 37:215-231

Sameer HA and Ali HMA. 2018. Effect of water extract on leaves of the plant in microorganisms causing gum inflammation and teeth and diagnosed PCR in the holy province of Karbala. Journal of Pharmaceutical Sciences and Research 10(7):1792-1794

Simon LD, Ogunwolu EO \& Okoroafor E. 2018. Impact of insect infestation on plant damage and yield of roselle (Hibiscus sabdariffa L.) in Benue State, Nigeria. International Journal of Plant and Soil Science 26(1):1-10

Sisk TD, Lanner AE, Switky AR \& Ehrlich PR. 1994. Identifying extinction threats: global analysis of the distribution of biodiversity and the expansion of the human enterprise. Bioscience 44:552-604

Zaime A and Gautier JY. 1989. Comparison of the diets of three sympathetic species of Gerbillidae in the saharan environment in Morocco. Review Ecology 44(3):263-278 